\title{
Technique for In-situ Meso-Scale Uniaxial Mechanical Testing in the SEM
}

\author{
Charles Spellman ${ }^{1}$, Vishaal Verma ${ }^{1}$, Alex Arzoumanidis, and Zachary Zanzinger ${ }^{1}$ \\ 1. Psylotech, Inc., Evanston IL, USA
}

Satisfying the varied demands of a versatile mechanical testing lab requires dynamic range in force measurement, positional control, and testing speeds. Furthermore, many labs are outfitted with numerous types of optical microscopes and other advanced imaging systems that need to be accommodated by insitu testing methods. Using a novel actuator architecture based on the Psylotech $\mu$ TS platform, the authors present a technique using precision machinery and industrial controllers to achieve: 25 nanometer crosshead control, $5 \mathrm{kN}$ axial force, and up to $35 \mathrm{~mm} / \mathrm{sec}$ at $600 \mathrm{~mm} / \mathrm{sec}^{2}$. The system is specifically suited for digital image correlation (DIC), an image processing technique capable of tracking displacement down to 0.02 pixels [1]. The load frame's inherently planar design and attention to limiting out of plane motion results in stable preservation of a specimen's area of interest within the imaging focal plane. With low out-gassing materials and vacuum chamber feed-through compatibility, the system is meant for SEM chambers; however, it is also superbly capable in air when operating under optical, confocal, or $\mu \mathrm{CT}$ imaging systems. The authors present an overview of the design constraints, system evolution, and performance specifications.

An SEM compatible load frame must handle high vacuum conditions in the region of $10^{-5}$ to $10^{-7}$ Torr. Additionally, most SEM stages are specified to handle up to 500 grams of mass, though in practice can handle up to $2 \mathrm{~kg}$ and still be translated with enough precision to enable the type of tests performed with mechanical load frames. While a large range of chamber volumes and configurations exist, it was found that a footprint of roughly $100 \mathrm{~mm}$ x $180 \mathrm{~mm}$ would satisfy the medium and larger size chambers. Also constraining the design was forcing the center of the specimen to be no more than $110 \mathrm{~mm}$ from one end of the load frame (in the axial direction) and as close in z-position to the electrode tip as possible. The first application required testing of metallic superalloy dogbone specimens up to $4.5 \mathrm{kN}$; these specimens were approximately $20 \mathrm{~mm}$ gauge length and required up to $50 \%$ strain. Quasi-static strain rates were required for these tests. The system was also expected to be used with optical microscopy, and needed a method to keep an area of interest centered under the optical or electron microscope objective (which is provided through a secondary centering stage). Less than 2 microns of out of plane motion and 1 micron displacement resolution were required to perform the test and in order to prevent false strains in DIC.

To satisfy these varied requirements, the $\mu$ TS platform was used as a starting point. The $\mu$ TS is a welltested actuator design encompassing a pre-loaded ballscrew and direct-drive servomotor actuator. The use of high efficiency permanent magnet motors along with the direct-drive architecture allows for high force capacity in a compact form. Additional benefits include high speed capability due to lack of a gearbox. The $\mu$ TS also incorporates Psylotech's proprietary high resolution sensor technology, enabling tests of over 6 orders of magnitude in force and displacement range. The 8 orders of magnitude in speed were less relevant to this quasi-static application. While the stock $\mu \mathrm{TS}$ does have a vacuum rating option, it only has $1.6 \mathrm{kN}$ of force capacity in this minimized configuration.

The primary modifications made to accommodate the above system requirements were to replace the 1 $\mathrm{mm}$ pitch ball screw actuator and bearing configuration with a $0.25 \mathrm{~mm}$ pitch roller screw actuator and $\mathrm{x}$ type bearings. This allowed the system to maintain its small size, but add about 4 times the load capacity 
due to a finer pitch. One potential drawback of the actuator is the possibility of backlash, since the roller screw and bearing are not preloaded. The backlash was found to be minimal in testing - not measurable within our optical displacement resolution when lightly loaded. Out of plane motion, minimized through the use of precision components and on-axis loading, was found to be less than \pm 2 microns at the max load tested of $3 \mathrm{kN}$ (Figure 2). Low out of plane motion improves DIC performance in the SEM, and also maximizes DIC potential from optical microscopy. Validated in the latter technique, $250 \mathrm{~nm}$ features could be resolved moving as little as $1 \mathrm{~nm}$ when the full field is averaged.

The system has applications from thick metals to single fibers. It can be outfitted with a motor controlled $\mathrm{y}-\mathrm{z}$ positioning stage on the crosshead for careful alignment of specimens in the SEM. Nanometer-level motion control (Figure 3) allows for tests ranging from micropillars to macroscale composites. NIST traceable load cells and compliance corrected encoder readings give trust in output data, and is often complemented with DIC to further enhance local strainfield measurement capability. The system has met the testing goals of the initial application, and satisfies myriad other testing methods as well [2].

\section{References:}

[1] Sutton et al, Experimental Mechanics 47(6) (2007), p. 789.

[2] The authors would like to thank Professor Michael Sutton of University of South Carolina for helping to mold the design and lending his expertise with DIC and SEM testing.

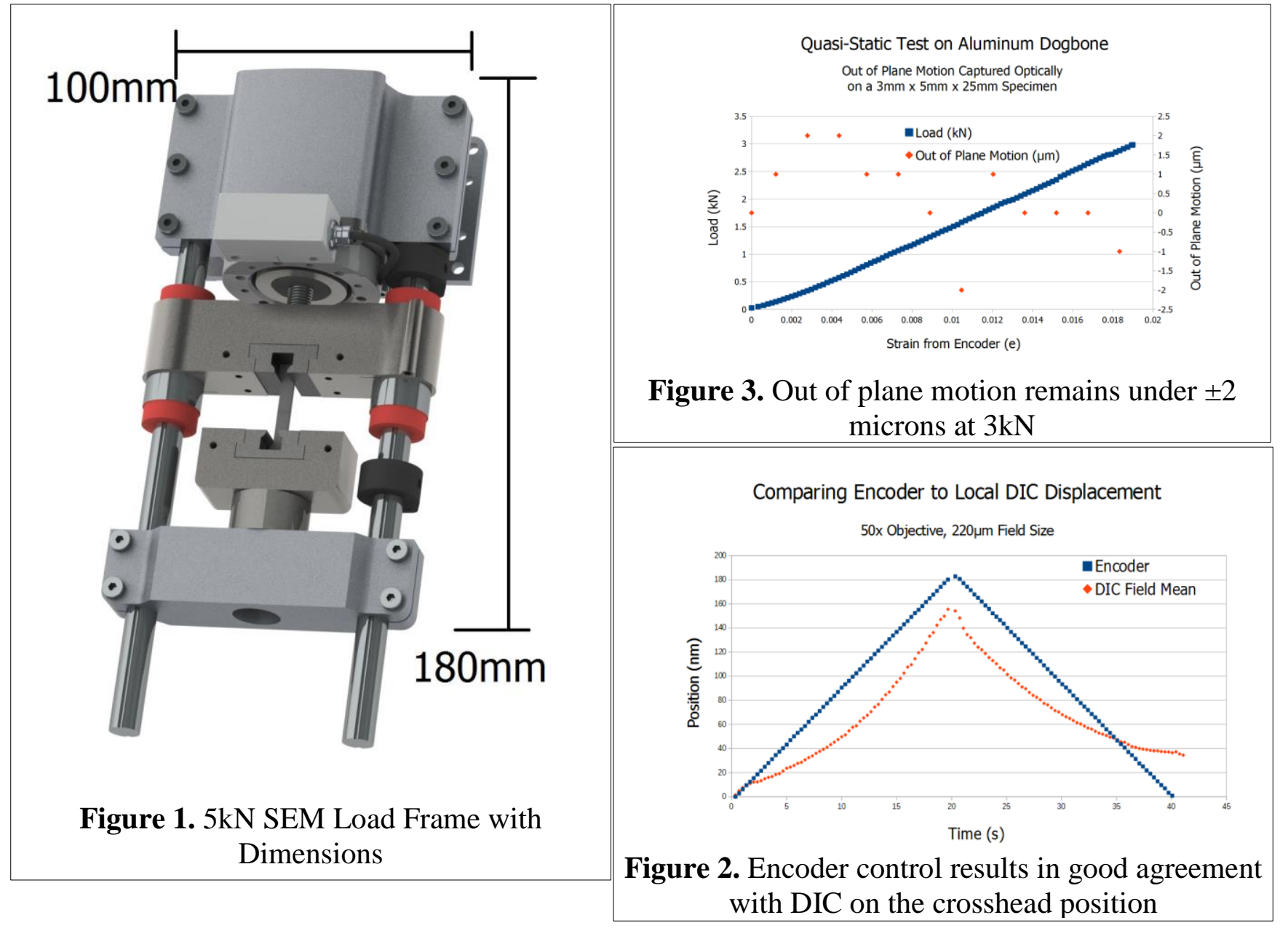

